\title{
Zukunft Integrierte Versorgung: die Kultur des Gemeinsamen
}

\author{
Angesichts der zunehmenden Komplexität der Patientenbetreuung erstaunt es \\ kaum, wie wenig die eine medizinische Berufsgruppe von den anderen weiss: von \\ deren Ausbildung, Kompetenzen oder Ressourcen. Dabei gäbe es viel zu entdecken \\ und zu nutzen bei den anderen - ein Plädoyer für mehr Denken und Handeln in \\ Netzwerken statt in Hierarchien.
}

Vorstand des Forum Managed Care (fmc)
Korrespondenz: PD Dr. Peter Berchtold Freiburgstrasse 41 CH-3010 Bern

\section{peter.berchtold[at]} college-m.ch
Medizin und Pflege haben sich in den letzten Jahrzehnten ausserordentlich differenziert: Die Zahl der Facharzttitel hat sich vervielfacht, ebenso die Zahl der Kliniken und Institute an Universitäts- und Grossspitälern. Medizinische Spezialitäten teilen sich in Subspezialitäten, gleich wie die Pflege oder die Physiotherapie. Waren in den achtziger Jahren drei bis fünf Fachpersonen an der Behandlung eines Spitalpatienten beteiligt, sind es heute 30 bis 50 .

Doch die Spezialisierung und Fragmentierung der Gesundheits- bzw. Krankheitsversorgung hat gravierende potentielle Gefahren:

- Sinkende Behandlungsqualität: Die Vielzahl an Schnittstellen ist kaum mehr handhabbar - die nächste Fachperson weiss häufig nur lückenhaft, was die letzte oder vorletzte gemacht hat. Dieser Informationsverlust führt unweigerlich zu Überdiagnostik und Mehrfachbehandlungen. Zwar werden die einzelnen Behandlungsschritte qualitativ hochwertig ausgeführt. Doch weil sie mangelhaft aufeinander abgestimmt sind, drohen die Ergebnisse für den Patienten ungenügend zu sein.

- Weniger Patientensicherheit: Überdiagnostik und Mehrfachbehandlungen sind risikoreich, zum Beispiel weil Patienten übermässig Röntgenstrahlen ausgesetzt sind oder ein Medikament unnötig wechseln müssen.

- Verteuerung des Systems: Überdiagnostik und Mehrfachbehandlungen können doppelt kostentreibend sein - erstens weil Zusätzliches und potentiell Unnötiges gemacht wird, zweitens weil Komplikationen abermals Kosten verursachen.

Ein breit anerkannter Lösungsansatz für diese Gefahren ist die Integrierte Versorgung, also die strukturierte disziplinen- und sektorenübergreifende Zusammenarbeit. Oder auf einen Nenner gebracht: mehr Kommunikation, mehr Koordination, mehr Kooperation. Mit einer solchen Kultur des Gemeinsamen werden Betreuungsqualität und Patientensicherheit gewährleistet - was letztlich die Kosten günstig beeinflusst. Die Evidenz dafür ist inzwischen erheblich.
Doch im Alltag ist diese Kultur des Gemeinsamen erst schwach entwickelt. Weil die Akteure - Leistungserbringer, Kostenträger, Patienten, Politik - sich in unterschiedlichen Realitäten mit unterschiedlichen Aufgaben, Ansprüchen und Standpunkten bewegen. Zwar halten alle die gleiche Landkarte des Schweizer Gesundheitswesens in den Händen. So wie unterschiedliche Berggänger eine Karte des gleichen Gebietes vor sich haben. Die Inhalte variieren aber von Gruppe zu Gruppe: Bei den Wanderern sind nur die Wanderwege eingezeichnet, bei den Bikern nur die Bike-Routen, bei den Bergsteigern nur die Klettersteige, bei den Geologen nur die Höhlen. Und während die einen mit Karten im Massstab 1:25000 unterwegs sind, haben andere Karten im Massstab 1:300000. Kurzum: Jeder kennt die eigene Realität, kann sich in dieser gut bewegen - und ist in den anderen häufig verloren.

\section{Die Spezialisierung und Fragmen- tierung der Gesundheits- bzw. Krankheitsversorgung hat gravie- rende potentielle Gefahren.}

Dabei gäbe es für alle so viel zu entdecken: Der Wanderer könnte via Klettersteig ein völlig neues Panorama erleben oder der Bergsteiger einen Höhlensee erkunden. Das heisst: Es braucht die Bereitschaft zum Perspektivenwechsel, die Offenheit, die Realität des anderen wahrzunehmen, und das Interesse, diese selber zu erkunden. Mit dem Ziel, eine gemeinsame Realität zu erlangen.

Wie notwendig solche Perspektivenwechsel sind, beschrieb der britische Ethnologe Nigel Barley in seinem Buch «Traumatische Tropen, Notizen aus meiner Lehmhütte» auf humorvolle Weise (die Szene spielt kurz vor seiner Abreise nach Afrika): «Der Augenblick für letzte Ratschläge war gekommen. Meine 
Familie, die bar jeden ethnologischen Sachverstands war, wusste nur, dass ich bekloppt genug war, ins Land der Wilden zu gehen, wo ich in ständiger Furcht vor Löwen und Schlangen im Urwald leben und froh sein musste, wenn ich nicht im Kochtopf landete. Es war mir eine gewisse Genugtuung, dass, als ich von den Dowayos wieder Abschied nahm, der Häuptling des Dorfs, in dem ich mich aufgehalten hatte, erklärte, er würde mich liebend gern zu meinem Dorf in England zurückbegleiten, wenn er nicht Angst vor einem Land hätte, in dem es immer kalt sei, in dem reissende Tiere von der Art der europäischen Hunde in der Missionsstation lebten und in dem es, wie bekannt, Menschenfresser gebe.»

Perspektivenwechsel und Kultur des Gemeinsamen sind die Grundlagen der Integrierten Versorgung. Denn Integrierte Versorgung bedeutet: Arbeiten in Netzwerken. Dadurch entsteht eine gemeinsame Realität, eine gemeinsame Sicht auf den Patienten. Vor allem dadurch können Über-, Unter- oder Fehlversorgung entdeckt und vermieden werden.

Die Kultur des Gemeinsamen verfolgt eine Reihe von wesentlichen Zielsetzungen:

- Alle sehen das Wohl des Patienten als oberste Aufgabe ihres Tuns.

- Alle stellen die eigenen Interessen unter dieses Gesamtinteresse.

- Alle (an-)erkennen die eigenen Grenzen und entwickeln die Einsicht, dass es immer etwas gibt, das ein anderer besser kann

- Alle sind bereit, Standes-, Hierarchie- und Konkurrenzdenken zu überwinden, und begegnen den anderen auf Augenhöhe.

Offenkundig muss eine solche Kultur des Gemeinsamen vielen Widerständen trotzen. Gleichwohl wächst die Erkenntnis in allen Bereichen, diese Kultur Schritt für Schritt zu entwickeln. Mögliche Mittel sind zum Beispiel:

- Interdisziplinäre Aus- und Weiterbildungen in integrativer Zusammenarbeit;

- Multidisziplinäre Teams, zum Beispiel bei der Behandlungsplanung oder in Qualitätszirkeln;

- Strukturierter Informations- und Datenaustausch im Netzwerk, am besten elektronisch (eHealth);

- Förderung der Patientenkompetenz, damit die Patienten mehr Eigenverantwortung entwickeln und ihren Betreuungspersonen auf Augenhöhe begegnen.

Der Integrierten Versorgung gehört die Zukunft, und sie verlangt von allen Beteiligten, ihr Koordinatensystem neu zu justieren: Netzwerke statt Hierarchien, Gesamtwohl des Patienten vor Eigeninteressen, flexibles (Aus-)Handeln statt starres Verordnen, Demut vor Übermut - nur so gedeiht eine Kultur des Gemeinsamen.

\section{fmc-Symposium 2013 am 27. Juni} im Hallenstadion Zürich

Das Symposium 2013 des Forum Managed Care fmc steht unter dem Titel «Zukunft Integrierte Versorgung: Die Kultur des Gemeinsamen». Die zentrale Frage lautet: Wie können die vielschichtigen Bedürfnisse und verschiedenen Wertesysteme der involvierten Akteure auf ein gemeinsames Ziel formiert werden: die bestmögliche Versorgung eines Patienten, einer Patientin? Das laufend aktualisierte Programm mit der Möglichkeit zur Anmeldung finden Sie unter www.fmc.ch/symposium.

\section{fmc-Förderpreis 2013}

Das fmc verleiht am 27. Juni 2013 den Förderpreis für herausragende Leistungen zur Entwicklung und Umsetzung der Integrierten Versorgung im Schweizer Gesundheitswesen. Die Preissumme beträgt 10000 Franken. Der Strategische Beirat des FMC entscheidet über die Vergabe des Förderpreises.

Teilnahmebedingungen: Alle zum Symposium 2013 eingereichten Projekte und Studien, die ganz oder hauptsächlich in der Schweiz bearbeitet wurden, sind für den Förderpreis angemeldet. Die Bewertung der Arbeiten erfolgt nach den Kriterien:

- Bezug zu den Themen des Symposiums: die Kultur des Gemeinsamen, insbesondere Zusammenspiel von Organisation, Prozessen und medizinischer Versorgung

- Nachweis des Nutzens von Integration und Vernetzung auf die medizinische Qualität und/oder die Wirtschaftlichkeit

Call for Abstracts und Einreichen der Projekte: Das fmc lädt alle Interessierten ein, am Symposium vom 27. Juni 2013 ihre wissenschaftlichen Studien, Optimierungsprojekte, Initiativen oder Innovationen in Form von Präsentationen oder interaktiven Workshops vorzustellen. Die Arbeiten sollen die Entwicklung, den Aufbau oder die Evaluation von innovativen Ansätzen zur Integrierten Versorgung darstellen und entsprechende Resultate aufweisen. Das Formular zum Einreichen Ihrer Projekt-Zusammenfassung finden Sie unter www.fmc.ch/symposium. ( $\rightarrow$ Jetzt einreichen).

Eingabeschluss ist der 31. März 2013. 